\title{
Vertical similarity in spoken word recognition: Multiple lexical activation, individual differences, and the role of sentence context
}

\author{
CYNTHIA M. CONNINE and DAWN G. BLASKO \\ State University of New York, Binghamton, New York \\ and \\ JIAN WANG \\ Hangzhou University, Hangzhou, China
}

\begin{abstract}
Four experiments investigated acoustic-phonetic similarity in the mapping process between the speech signal and lexical representations (vertical similarity). Auditory stimuli were used where ambiguous initial phonemes rendered a phoneme sequence lexically ambiguous (perceptual-lexical ambiguities). A cross-modal priming paradigm (Experiments 1, 2, and 3) showed facilitation for targets related to both interpretations of the ambiguities, indicating multiple activation. Experiment 4 investigated individual differences and the role of sentence context in vertical similarity mapping. The results support a model where spoken word recognition proceeds via goodness-of-fit mapping between speech and lexical representations that is not influenced by sentence context.
\end{abstract}

In order to understand spoken language, the information in the speech signal must be mapped onto an internal representation that supports comprehension. The mapping process from the physical signal to a lexical representation is not a trivial process; typical situations in which individuals produce and comprehend spoken language include background noise, mispronunciations, misarticulations, phonological variations, and segment reductions. The focus of the present experiments is on how the speech signal maps onto units that support word recognition. The central hypothesis investigated in the experiments is that lexical activation proceeds via a process of vertical similarity mapping. A central aspect of vertical similarity mapping is that a lexical representation is activated as a consequence of the success of the mapping process from the speech signal to a lexical representation. The success of vertical similarity mapping is modulated by the similarity of the signal to a stored representation. Of particular interest in the present experiments is the mapping process for word-initial phonemes and their role in permitting multiple lexical activation.

Portions of the data reported in this paper were presented at the 1990 meeting of the Psychonomic Society in New Orleans. This research was supported by NIDCD Grant R29 NS26587 to the first author. Additional support was provided by the Center for Cognitive and Psycholinguistic Science. Thanks go to Joanne Miller and three anonymous reviewers for their comments on the manuscript, and to Debra Titone, Cara Schmitt, and Stacey Garsson for their assistance in running subjects. Correspondence should be addressed to $\mathrm{C}$. Connine, State University of New York at Binghamton, Department of Psychology, Binghamton, NY 13901 (e-mail: connine@bingvmb.bitnet).
Vertical similarity mapping is a process that forms a basis for many theoretical accounts of spoken word recognition. A critical distinction among models is the degree of specification required for successful lexical activation as a function of sequential position. At one extreme, one version of the cohort model requires a complete mapping from the speech signal to a lexical representation (Marslen-Wilson \& Welsh, 1978). Crucial to this view is a successful mapping of word-initial information to a lexical representation, since this portion of the word defines the set of lexical candidates. Successive phonemes serve to rule out lexical hypotheses based on a successful match of initial phonemes. A more recent version of the cohort model (Marslen-Wilson, 1987) weakened the reliance on a complete mapping for word-initial phonemes. Here, mismatching information between the input and a lexical representation (at the level of phonetic features) will not preclude activation (but see Marslen-Wilson \& Zwitserlood, 1989, for a reemphasis on the importance of word onsets). This claim highlights one way in which similarity mapping may be probabilistic - specifically, there may be a partial match between a fully specified segment in the speech signal and a lexical representation. Connine, Blasko, and Titone (1993) have shown that the degree of partially matching information in a fully specified segment determines the success of lexical activation (see also Connine, 1994; Connine, Blasko, \& Titone, 1992; Connine, Blasko, Titone, \& Ferriby, 1993; Connine, Titone, \& Blasko, 1991; Connine, Titone, \& Ferriby, 1993). Using a semantic cross-modal priming paradigm, Connine, 
Blasko, and Titone (1993) showed that lexical decisions to an associate to a base word (e.g., SERVICE) were facilitated when a nonword was presented that contained an initial segment differing in one linguistic feature from the base word (e.g., zERVICE). Stated somewhat differently, a small degree of mismatching information did not preclude activation. No facilitation for associates to a base word was found when a nonword was presented with an initial segment that differed by many linguistic features (e.g., GERVICE). These results demonstrate that a partial match between the speech and a lexical representation is sufficient for initial lexical activation (Connine, 1994; Marslen-Wilson \& Zwitserlood, 1989; Milburg, Blumstein, \& Dworetzky, 1988). Furthermore, the probability of activating a particular lexical entry decreases as the degree of similarity between the speech input and a lexical representation decreases. Evidence that partially matching information in a fully specified segment can activate a lexical representation is also consistent with another major model of spoken word recognition, the TRACE model (McClelland \& Elman, 1986). One recent instantiation of a connectionist model of this class is the recurrent net model outlined by Norris (1990). Norris provides a simulation that models the results of Connine, Blasko, and Titone (1993) exactly in that activation of lexical candidates was a function of vertical similarity mapping.

A second dimension of vertical similarity, particularly relevant for the present experiments, is that the speech signal may consist of an incomplete specification of the acoustic-phonetic information. One such case is a component speech sound in a word that is perceptually ambiguous. An ambiguous segment (such as a token from a speech series taken from the category boundary region) will result in more than one phonetic category that is consistent with the input. Category ambiguity has been accommodated in a number of models of speech perception. In early feature detector models, ambiguous tokens were assumed to activate an overlapping region of sensitivity of two detectors (see Eimas \& Corbit, 1973). In more recent accounts, the representation of phonemes consists of a graded structure in which some tokens are better or more prototypical category instances than other tokens (see Miller, Connine, Shermer, \& Kluender, 1983; Miller \& Volaitis, 1989; and Samuel, 1982, for evidence concerning the graded representation of phonemes). Ambiguous tokens are consistent with two possible speech categories and are poor instantiations of either category. Massaro (1987) offers one explicit mathematical account for representing graded category structure that relies on concepts from fuzzy logic.

Experiments 1, 2, and 3 were designed to investigate the consequences of speech category ambiguity for spoken word recognition. One goal of the experiments was to determine the consequences of incomplete phoneme specifications for lexical activation. The experiments used stimuli where an initial ambiguous phoneme renders the sequence ambiguous at the lexical level (perceptual- lexical ambiguity). An example of a perceptual-lexical ambiguity is a sequence in which an initial phoneme is ambiguous between $a / d /$ and $a / t /$ when it precedes the sequence ENT. Two potential interpretations result: one interpretation where the initial phoneme is assigned a voiced value (DENT), and one interpretation where the initial phoneme is assigned a voiceless value (TENT). The vertical similarity-mapping hypothesis predicts that lexical activation may not be compromised since partially matching information in an ambiguous segment will be sufficient to permit successful mapping. Perceptuallexical ambiguities provide a strong test of the similaritymapping hypothesis because they permit an assessment of multiple lexical activation given comparable degrees of similarity with the input.

Previous research has used perceptual-lexical ambiguities in speech identification experiments in order to investigate the influence of sentence context on speech perception (Connine, 1987; Garnes \& Bond, 1976). These experiments have demonstrated that identification of ambiguous tokens is influenced by a preceding sentence context such that the identification response forms a word consistent with the semantic bias of the sentence. Of particular relevance are the results of Connine (1987), who found comparable reaction times for context-consistent and context-inconsistent identifications of ambiguous tokens. These results were taken as support for a model where context was used to select among two competing hypotheses. Stated somewhat differently, contextual information was used neither to preselect potential lexical candidates nor to directly influence the way the acoustic information was interpreted (see Samuel, 1981, for a similar claim concerning the relationship between sentence level representations and speech processing). Rather, the perceptually ambiguous speech was assumed to map onto two compatible and comparably available lexical hypotheses.

Experiments 1, 2, and 3 were designed to provide converging evidence for the claim that perceptual-lexical ambiguities succeed in activating compatible lexical representations. A cross-modal priming paradigm was used in which perceptual-lexical ambiguities were presented and followed by visual targets that were semantically related to either the voiced or the voiceless interpretation. The cross-modal priming paradigm is ideal for examining issues concerning the relationship between the integrity of the speech signal and spoken word recognition. It permits a direct assessment of multiple activation given a constant speech signal. This contrasts with the phoneme identification paradigm where multiple activation was inferred indirectly via consideration of identification responses and reaction times. In the crossmodal priming task, facilitation for lexical decisions to related visual targets (relative to a control) can be taken as evidence that a lexical representation was activated. To the extent that voiced- and voiceless-related targets are facilitated comparably, it will be possible to conclude that there was activation of multiple lexical hy- 
potheses. Experiments 1, 2, and 3 used the same basic cross-modal priming methodology with somewhat different control conditions.

Experiment 4 investigated two additional aspects of similarity mapping. First, individuals may vary in the degree that they are able to successfully utilize the relevant acoustic-phonetic information for lexical activation. Individual differences in these abilities have consequences for speech perception and spoken word recognition. Experiment 4 examined explicitly the contribution of individual differences. A second issue examined in Experiment 4 was the role of sentence context to auditory word recognition (see Fodor, 1983). Experiment 4 was designed to determine the way in which sentence context contributes to similarity mapping.

\section{EXPERIMENT 1}

Experiment 1 used a cross-modal priming methodology in which auditorily presented perceptual-lexical ambiguities were followed by visually presented targets. The visually presented targets were related to the voiced or voiceless interpretation of the perceptual-lexical ambiguity. Lexical decision reaction times for the related targets were compared with visually presented control targets that were unrelated to a given perceptual-lexical ambiguity.

\section{Method}

Subjects. A total of 68 subjects participated in the experiment in partial fulfillment of requirements for a psychology course.

Materials. Twenty pairs of one-syllable words ( 40 words total) were selected that differed only in the voicing value of the initial phoneme and were of comparable frequency (Francis \& KuCera, 1982). The average frequency of the voiced stimuli was $186 \mathrm{oc}-$ currences per million $(S D=287)$, and the average frequency of the voiceless stimuli was 155 occurrences per million $(S D=383)$. The 40 words were recorded in isolation on audiotape by a male speaker in a quiet room. The stimuli were low-pass filtered at $4.8 \mathrm{kHz}$ and digitized at $10 \mathrm{kHz}$ (12-bit resolution).

Perceptual-lexical ambiguities. In order to obtain perceptually ambiguous tokens of the stimuli, voicing continua were created from the naturally produced utterances of each word pair. In order to generate the voicing continuum for each word pair, successive portions of energy were excised from the target word starting with the voiced consonant, beginning at the onset of the burst of the voiced segment. The excised portions were replaced with equally long acoustic segments of energy taken from the voiceless word starting with the end of the closure for the voiceless segment (the onset of the release burst). The excised segments were cut at zero crossings. The durations of the replaced and excised portions were successively lengthened to create voicing continua. In order to determine which tokens were perceived as perceptually ambiguous, a pilot experiment was conducted in which the continua were presented to a group of 20 volunteer listeners for identification. These listeners did not participate in the cross-modal priming experiment or in generation of the related visual targets. Identification functions were used to select the stimulus for a given continuum that was ambiguous (approximately $50 \%$ of the time, the stimulus was identified as voiced, and $50 \%$ of the time, the stimulus was identified as voiceless). Appendix A shows the voicing stimulus values and the identification functions for each con- tinuum. The VOT values for the stimuli used in the cross-modal priming experiment are underlined. In an attempt to increase the degree of perceptual ambiguity of the stimuli, noise was added to half of the perceptual-lexical ambiguities (the boundary stimuli). For the noise-added stimuli, the extent of the VOT that defined the boundary stimulus was altered such that voicing information alternated with amplitude-matched white noise in 5 -msec steps. The replacement procedure began with the first $5 \mathrm{msec}$ of the stimulus (beginning with the onset of the burst) and continued throughout the VOT duration for a given stimulus.

Visual targets. Selection of the appropriate semantically related targets was based on the responses obtained by presenting the list of 40 stimuli to a group of 30 volunteer subjects. These subjects did not participate in the cross-modal priming experiment. They were instructed to generate a related word for each stimulus. Selection of the related visual targets was based on subjects' responses using a criterion of greater than $50 \%$ agreement. Unrelated control visual targets were selected that were comparable in length (number of letters), morphological complexity, and Francis and Kuxera (1982) word frequency (see Appendix A). The average frequency for the voiced-related targets was 173 occurrences per million ( $S D=170$ ), and the average frequency for the voiceless-related targets was 212 occurrences per million $(S D=$ 173). The average frequency for the voiced control targets was 183 occurrences per million $(S D=249)$, and the average frequency for the voiceless control targets was 193 occurrences per million $(S D=183)$.

Two preliminary experiments were conducted prior to the main experiment with the perceptual-lexical ambiguities. First, the visual targets were presented in isolation to a group of 20 subjects in a lexical decision task. An additional 20 nonwords were included. Overall, there was no difference between the related and control targets [678 and $687 \mathrm{msec}$, respectively; $t(19)=1.15, p=.26$ ]. A second preliminary experiment was conducted in order to demonstrate that the visual targets produced a priming effect for the unambiguous auditory stimuli. A total of 20 new subjects were presented with the unambiguous auditory versions of the stimuli. Two lists were prepared in which each experimental stimulus was presented once followed by a visual target. The visual targets were presented at the offset of the auditory stimulus. The subjects were instructed to perform a lexical decision to the visual target. Related and unrelated targets were counterbalanced across the two lists. A comparable number of auditory filler words were selected. Filler words were used to present nonword visual targets. Visual nonword targets were created by altering one letter of a real word. As expected, a twoway analysis of variance (ANOVA) on the items means with interpretation (voiced vs. voiceless) and target type (related vs. control) showed a robust priming effect $[95 \mathrm{msec}$; i.e., 587 vs. $682 \mathrm{msec}$, related vs. control, respectively; $F(1,19)=106$, $\left.M S_{\mathrm{e}}=2,293, p<.01\right]$. Also significant was the two-way interaction of interpretation and target type $[F(1,19)=23.9$, $\left.M S_{\mathrm{e}}=1,593, p<.01\right]$. Overall, the voiced interpretation resulted in a substantially larger priming effect (133 msec; i.e., $567 \mathrm{vs.}$ $700 \mathrm{msec}$, related vs. control) than the voiceless interpretation ( $57 \mathrm{msec}$; i.e., 607 vs. $664 \mathrm{msec}$, related vs. control).

Procedure. On the basis of the results of the two preliminary experiments, four lists were created that contained the 20 perceptuallexical ambiguities. Within each list, each perceptual-lexical ambiguity was presented once. The voiced-related visual target, the voiced-control visual target, the voiceless-related visual target, and the voiceless-cont rol visual target were distributed across the four lists such that no visual target was presented more than once. Relatedness and voiced/voiceless-related visual targets were counterbalanced across lists. This design ensured that no subject heard a perceptual-lexical ambiguity more than once or saw a given visual target more than once. An additional set of 20 unambiguous auditory stimuli were included in each list for presentation with 
the nonword visual targets. The subjects were tested individually or in groups of two and were randomly assigned to one of the four lists. Stimuli were stored on disk, and presentation was controlled by a microcomputer. The subjects heard the stimuli over headphones at a comfortable listening level in a sound-treated room. A timing mark, inaudible to the subject, was placed coincident with the offset of each stimulus. The timing mark served to trigger presentation of the visual target. The subjects' responses and lexical decisions were recorded. Trials were separated by $2 \mathrm{sec}$ of silence, and the subjects were given a rest break after 50 trials. The subjects were instructed to listen to the auditory stimuli and to identify each visual target as a word or nonword quickly and accurately. They indicated their responses by pressing an appropriately labeled response key (word or nonword). Prior to the experimental trials, a set of 10 practice trials was presented.

\section{Results and Discussion}

Table 1 shows lexical decision times as a function of target type (related vs. control) and voiced versus voiceless interpretation. In all experiments, error reaction times were excluded from all of the analyses.

Overall, there was a 44-msec advantage for related targets relative to control targets $(668 \mathrm{vs.} 712 \mathrm{msec}$, respectively). As is indicated in Table 1, the priming effect was larger when activation of the voiced-related interpretation was assessed relative to when activation of the voiceless-related interpretation was assessed (60 vs. $29 \mathrm{msec}$ ). The asymmetry in the size of the priming effect for the ambiguous stimuli is similar to that found for the unambiguous stimuli in the preliminary experiment. It should also be noted that the size of the priming effect for ambiguous stimuli was reduced relative to that for the unambiguous stimuli. This suggests that the ambiguous stimuli were less effective in activating a lexical representation than were the unambiguous stimuli.

A two-way ANOVA with relatedness (related vs. control) and interpretation (voiced vs. voiceless) was conducted on lexical decision times. ${ }^{1}$ Variability was accessed across subjects $(F 1)$ and across items $(F 2)$ in all analyses. The ANOVA showed that the main effect of relatedness was significant $\left[F 1(1,67)=34, M S_{\mathrm{e}}=4,004\right.$, $\left.p<.01 ; F 2(1,19)=16.7, M S_{\mathrm{e}}=2,316, p<.01\right]$. Also significant was the main effect of interpretation $[F 1(1,67)=$ $22, M S_{\mathrm{e}}=5,788, p<.01 ; F 2(1,19)=5.5, M S_{\mathrm{e}}=7,006$, $p<.05]$. Overall, reaction times were faster for the voiced interpretation than for the voiceless interpretation, regardless of relatedness ( $668 \mathrm{vs} .712 \mathrm{msec}$, respectively). The interaction of relatedness and interpretation was marginally significant across subjects $[F 1(1,67)=3.34$, $\left.M S_{\mathrm{e}}=4,868, p=.068\right]$ but missed significance across items $\left[F 2(1,19)=1.8, M S_{\mathrm{e}}=2,353, p=.18\right]$. A com-

Table 1

Lexical Decision Reaction Time (in Milliseconds) and Percent Correct for Related and Control Targets as a Function of Voiced Versus Voiceless Interpretation in Experiment 1

\begin{tabular}{lccccc}
\hline & \multicolumn{2}{c}{ Related } & & \multicolumn{2}{c}{ Control } \\
\cline { 2 - 3 } \cline { 5 - 6 } Interpretation & RT & \% Correct & & RT & $\%$ Correct \\
\hline Voiced & 638 & 96 & 698 & 95 \\
Voiceless & 697 & 98 & & 726 & 95 \\
\hline
\end{tabular}

parable ANOVA on percentage correct responses showed slightly better performance on related targets $(97 \%$ correct) than on control targets $(95 \%)[F 1(1,67)=5.7$, $\left.M S_{\mathrm{e}}=77, p<.05 ; F 2(1,19)=5.7, M S_{\mathrm{e}}=17, p<.05\right]$. No other effects were significant.

The results of Experiment 1 provide tentative evidence for two major conclusions. First, the results demonstrate that a perceptually ambiguous word-initial phoneme does not preclude lexical activation. Second, multiple lexical hypotheses (in this case, two lexical hypotheses) that are equivalently compatible with the acoustic-phonetic input may be activated.

\section{EXPERIMENT 2}

The purpose of Experiment 2 was to replicate the results of Experiment 1 using a slightly different version of the cross-modal priming paradigm. Some versions of the paradigm employ auditory control stimuli instead of visual control stimuli (as were used in Experiment 1). In the auditory control version of the paradigm, an auditory control word is selected for each auditory experimental word that is matched on a number of dimensions (length, syllable structure, lexical frequency) but differs in terms of acoustic-phonetic structure. The visual target that is selected as a semantic associate to the experimental auditory stimulus is presented following the control and experimental auditory stimuli. The auditory control design has some advantages over the visual control design since lexical decision reaction times for the same visual target are compared. One simply manipulates the nature of the auditory stimulus, and the visual target is identical in the related and unrelated conditions. This design eliminates possible contributions from unintended differences in related and control visual targets to any observed priming effect. Experiment 2 used the set of perceptual-lexical ambiguities used in Experiment 1 and matched auditory controls in a cross-modal priming experiment.

\section{Method}

Subjects. A group of 124 subjects participated in the experiment either for credit in an introductory psychology class or for pay.

Materials. The set of 20 perceptual-lexical ambiguities used in Experiment 1 were used in Experiment 2. An auditory control was selected for each perceptual-lexical ambiguity that differed in acoustic-phonetic structure but was matched in terms of lexical frequency and length (see Appendix B). The average frequency of occurrence of the voiced auditory controls was 127 occurrences per million $(S D=176)$, and the average frequency of the voiceless auditory controls was 216 occurrences per million $(S D=$ 156). A total of 40 auditory filler words (the 20 auditory filler words used in Experiment 1 along with 20 additional comparable fillers) were included. The additional filler words were necessary in order to maintain comparable numbers of word and nonword visual targets. Visual nonword targets were constructed for the 20 additional filler words. The 40 auditory controls and 20 additional auditory fillers were recorded in isolation on audiotape by the same male speaker used to record the perceptual-lexical ambiguities. Each auditory control word was low-pass filtered at $4.8 \mathrm{kHz}$, 
digitized at $10 \mathrm{kHz}$ (12-bit resolution), and stored on disk for presentation.

Procedure. Four lists of stimuli were prepared. Each list contained one presentation of the 20 perceptual-lexical ambiguities, 20 matched auditory controls, and 40 filler words. Across the four lists, the voiced-interpretation visual target was presented either following the appropriate perceptual-lexical ambiguity or following its matched auditory control; the voiceless-interpretation visual target was presented either following the appropriate perceptual-lexical ambiguity or following its matched auditory control. Voiced-related and voiceless-related visual targets were counterbalanced across the four lists, and no subject performed a lexical decision on a visual target more than once. The subjects were randomly assigned to one of the four lists. All other aspects of stimulus presentation were identical to those of Experiment 1.

\section{Results and Discussion}

Table 2 shows lexical decision reaction time as a function of voiced or voiceless interpretation and auditory stimulus (perceptual-lexical ambiguity or auditory control).

Overall, lexical decision reaction times were faster $(18 \mathrm{msec})$ when the visual target was related to a possible interpretation of the auditory input (when it followed a perceptual-lexical ambiguity) than when the visual target was not related to the auditory input (when it followed the auditory control) (642 vs. $660 \mathrm{msec}$, respectively). The relative size of the related-target advantage was comparable for the voiced and voiceless interpretations (16 and $20 \mathrm{msec}$, respectively). A two-way ANOVA with target relatedness (voiced vs. voiceless) and auditory stimulus type (auditory related vs. auditory control) showed a main effect of auditory stimulus type that was significant across subjects $[F 1(1,123)=9.7$, $\left.M S_{\mathrm{e}}=3,852, p<.01\right]$ but missed significance across items $\left[F 2(1,19)=2.07, M S_{\mathrm{e}}=2,266, p=.16\right]$. The main effect of target relatedness was significant, indicating that voiceless-related targets were responded to faster than were voiced-related targets $[F 1(1,123)=$ $51.7, M S_{\mathrm{e}}=4,625, p<.01 ; F 2(1,19)=7.16, M S_{\mathrm{e}}=$ $5,669, p<.01]$. No other effects were significant. Comparable ANOVAs on percentage correct responses showed no significant difference between the auditory related $(97 \%$ correct) and auditory control $(97 \%$ correct) (all $\left.F_{\mathbf{s}}<1\right)$. No other effects were significant.

The results of Experiment 2, which used a slightly different methodology, replicate the findings of Experiment 1 . Overall, lexical decision reaction times to visual

Table 2

Lexical Decision Reaction Times (in Milliseconds) and Percent Correct as a Function of Target Relatedness (Voiced vs. Voiceless) and the Auditory Stimulus (Related vs. Control) in Experiment 2

\begin{tabular}{|c|c|c|c|c|}
\hline \multirow[b]{3}{*}{ Target Relatedness } & \multicolumn{4}{|c|}{ Auditory Stimulus } \\
\hline & \multicolumn{2}{|c|}{ Related } & \multicolumn{2}{|c|}{ Control } \\
\hline & RT & $\%$ Correct & $\mathrm{RT}$ & $\%$ Correct \\
\hline Voiced & 665 & 97 & 681 & 96 \\
\hline Voiceless & 619 & 97 & 639 & 98 \\
\hline
\end{tabular}

targets were facilitated when they followed the perceptuallexical ambiguities relative to when they followed an unrelated auditory stimulus. The size of the priming effect in Experiment 1 was larger than that found in Experiment 2 ( 44 vs. $18 \mathrm{msec}$ ). This suggests that the priming effect found in Experiment 1 included a potential contribution from uncontrolled aspects of the visual targets. In contrast to that found in Experiment 1, the priming effect found in Experiment 2 was relatively symmetric for the voiced- and voiceless-related targets.

The results of Experiment 2 suggest that uncontrolled properties of the visual targets used in Experiment 1 contributed to the overall size of the priming effect as well as observed asymmetries in the voiced versus voiceless interpretations. Other factors may also have contributed to the size of the priming effect. In particular, one possible source of stimulus contribution in the auditory control version of the cross-modal priming paradigm was the relative ambiguity of the auditory control and the experimental stimuli. The experimental stimuli in our experiments (the perceptual-lexical ambiguities) were by their nature ambiguous, whereas the control auditory stimuli were (intentionally) unambiguous. This difference between the experimental and control stimuli may have contributed to lexical decision reaction times over and above any semantic priming effects. These considerations suggest an explanation for the reduction in the size of the priming effect in Experiment 2 relative to that in Experiment 1. The absence of perceptual ambiguity in the auditory controls may have contributed to a relatively faster response. Conversely, the presence of an ambiguity in the perceptual-lexical ambiguities could have potentially resulted in slower responses (regardless of the semantic relationship between the auditory and visual stimulus). This could effectively reduce the size of the priming effect, since the baseline reaction time reflected in the responses following the control auditory stimuli would be faster and the reaction time reflected in the responses following the perceptual-lexical ambiguities would be slower (all other things being equal).

Experiment 3 was designed to investigate this possibility by utilizing an auditory control more analogous to the perceptual-lexical ambiguities. This was accomplished by replacing the unambiguous auditory controls with auditory controls that began with an ambiguous phoneme taken from a voiced-voiceless continuum.

\section{EXPERIMENT 3}

\footnotetext{
Method

Subjects. A total of 104 subjects participated in the experiment for credit in an introduction to psychology course.

Materials. The 20 perceptual-lexical ambiguities used in Experiments 1 and 2 were used. An additional set of 20 word pairs that differed in the voicing value of the initial phoneme were selected to serve as controls (see Appendix B). The 20 pairs of words were selected to be as comparable as possible in frequency and in length to the perceptual-lexical ambiguities, but the limited number of stimuli available prevented precise matching. The average
} 
frequency of the voiced interpretation of the ambiguous auditory controls was 59 occurrences per million $(S D=93)$, and the average frequency of occurrence of the voiceless interpretation was 58 occurrences per million $(S D=90)$. The auditory controls were matched with perceptual-lexical ambiguities such that there was no semantic relationship between them. The 40 words were recorded in isolation by the same speaker used in the previous experiments, low-pass filtered at $4.8 \mathrm{kHz}$, and digitized at $10 \mathrm{kHz}$ (12-bit resolution). Voicing continua were created from the naturally produced speech using a waveform editor (for further details see the Materials section in Experiment 1). The 20 voicing continua were presented to 4 listeners, and the obtained identification functions along with experimenter intuitions were used to select a perceptually ambiguous stimulus (see Appendix B for the VOT values selected for each stimulus).

Procedure. The four lists used in Experiment 2 were modified such that the unambiguous auditory controls were replaced with ambiguous auditory controls. As in the previous experiments, no subject saw a visual target more than once, and voiced-related and voiceless-related targets were counterbalanced across the four lists. In addition, a set of filler items were used where 10 stimuli with word-initial ambiguous phonemes, 10 stimuli with wordinitial unambiguous phonemes, and 20 stimuli with word-initial unambiguous phonemes were followed by visual nonword targets. All other aspects of stimulus presentation were identical to those of Experiment 1.

\section{Results and Discussion}

Table 3 shows mean lexical decision reaction time as a function of auditory stimulus (ambiguous related and ambiguous control) and visual target relatedness (voiced vs. voiceless).

Overall, there was a $17-\mathrm{msec}$ priming effect (641 vs. $658 \mathrm{msec}$, ambiguous related vs. ambiguous control, respectively). A two-way ANOVA showed that the priming effect was significant across subjects $[F 1(1,103)=$ 8.6, $\left.M S_{\mathrm{e}}=3,350, p<.01\right]$ and marginally significant across items $\left[F 2(1,19)=3.5, M S_{\mathrm{e}}=1,525, p=.07\right]$. Also significant was the main effect of voiced versus voiceless interpretation $\left[F 1(1,103)=14.5, M S_{\mathrm{e}}=4,601, p<\right.$ $\left..01 ; F 2(1,19)=4.7, M S_{\mathrm{e}}=2,199, p<.01\right]$. No other effects were significant. A comparable ANOVA on the percentage correct response showed no difference between the related and control conditions (all $F \mathrm{~s}<1$ ).

The results of Experiment 3 provide a replication of Experiments 1 and 2. Taken together, the results suggest that acoustic-phonetic ambiguity at a words' onset does not preclude activation of lexical alternatives that are partially compatible with the input. Furthermore, the re-

Table 3

Lexical Decision Reaction Times (in Milliseconds) and Percent Correct as a Function of Target Relatedness (Voiced vs. Voiceless) and the Auditory Stimulus (Ambiguous Related vs. Ambiguous Control) in Experiment 3

\begin{tabular}{cccccc}
\hline & \multicolumn{3}{c}{ Auditory Stimulus } \\
\cline { 2 - 3 } & \multicolumn{2}{c}{$\begin{array}{c}\text { Ambiguous } \\
\text { Related }\end{array}$} & & \multicolumn{2}{c}{$\begin{array}{c}\text { Ambiguous } \\
\text { Control }\end{array}$} \\
\cline { 2 - 3 } \cline { 5 - 6 } Target Relatedness & RT & \% Correct & & RT & \% Correct \\
\hline Voiced & 657 & 95 & & 668 & 95 \\
Voiceless & 626 & 97 & & 649 & 97 \\
\hline
\end{tabular}

sults of Experiments 2 and 3 suggest that the alternative lexical interpretations (voiced and voiceless onsets) are activated to a comparable degree. In both experiments, the size of the priming effect for the voiced and voiceless alternatives was comparable. One possible concern about the results of Experiments 2 and 3 is that although the subject analyses showed robust priming effects, the item analyses were not robust in either experiment (Experiment 2, $<<.16$; Experiment 3,p<.07). In order to increase power in the item analyses, a post hoc ANOVA was conducted with experiment as a between-item factor (Experiment 2 and Experiment 3 ) and voicing interpretation and relatedness as within-item factors. The post hoc items analysis with increased power showed a robust priming effect $\left[F 2(1,38)=5.3, M S_{\mathrm{e}}=1,896\right.$, $p<.05]$.

\section{Individual Differences and Sentence Context Effects}

Across Experiments 1, 2, and 3, the data showed patterns of facilitation that suggested activation of both interpretations of the perceptual-lexical ambiguities. Despite somewhat different methodologies, the experiments revealed remarkably similar patterns of results. The systematicity provides strong support for the claim that the relative similarity of the input to a lexical representation modulates the mapping process between the speech signal and a lexical representation. Moreover, the experiments demonstrate that the integrity of word-initial phonemes is not critical for successful vertical similarity mapping (see Connine, Blasko, \& Titone, 1993).

Experiment 4 was designed to extend the results of Experiments 1, 2, and 3 in two directions. One purpose of Experiment 4 was to investigate the role of individual differences in similarity mapping. A second purpose was to investigate the influence of sentence context on multiple activation in perceptual-lexical ambiguities. Consider, first, the role of individual differences. In general, the contribution of individual differences to spoken language processing has received little attention, but some recent work demonstrated considerable individual variability in the consequences of reduced cue stimuli (e.g., a BAIT-DATE speech continuum in which the initial burst and/or formant cues to phoneme identity were removed) for phoneme identification performance (Hazan \& Rosen, 1991).

In a different domain, homonym processing, Gernsbacher and colleagues (Gernsbacher, 1990; Gernsbacher \& Faust, 1991) have identified individual differences in the ability to suppress an inappropriate meaning of a lexical ambiguity. In these experiments, lexical-ambiguity sentences (e.g., "He dug with the spade") and control sentences (e.g., "He dug with the shovel") were used, and subjects were asked to determine whether a target (e.g., ACE) was related to a just-read sentence. In both lexical-ambiguity and control sentences, the correct response is that the target is unrelated. Gernsbacher and 
Faust found that subjects were slower to judge ACE as unrelated in the lexical-ambiguity sentence relative to the control sentence when the target word was presented immediately after the sentence. The interference effect was attributed to activation of both interpretations of the lexical ambiguity. When presentation of the target was delayed, individual differences emerged in that interference effects were still evident for less skilled comprehenders but not for more skilled comprehenders. Gernsbacher and Faust interpreted these results as indicating that more skilled comprehenders are able to suppress contextually inappropriate interpretations of lexically ambiguous words more effectively.

In Experiment 4, individual differences were assessed in order to determine whether parallel differences emerge in subjects' abilities to suppress inappropriate interpretations of a perceptual-lexical ambiguity. A second goal of Experiment 4 was to investigate the contribution of sentence context to resolution of perceptual-lexical ambiguities. As previously described, the architectural relationship between sentence context and speech processing has been investigated by observing effects of sentence context on speech identification (Connine, 1987). It was argued that sentence context does not directly contribute to the interpretation of the acoustic signal. Rather, the data were consistent with a mechanism where a perceptuallexical ambiguity resulted in initial activation of multiple lexical hypotheses, and sentence context was used to select among them. Experiment 4 was designed to provide converging evidence for this claim. In the experiment, perceptual-lexical ambiguities were embedded in sentence contexts that were biased toward one of the alternative interpretations. This manipulation permitted an assessment of multiple activation and the role of sentence context in ambiguity resolution.

The design of Experiment 4 followed that of Gernsbacher and colleagues. Perceptual-lexical ambiguities differ from homonyms in that the stimulus input must map onto two lexical forms as well as two distinct lexical meanings. However, there are a number of advantages of using the same methodology as Gernsbacher and Faust (1991). One advantage is that it provides a means to identify commonalities and/or divergences in processing lexical and perceptual-lexical ambiguities. A second advantage is that the interference paradigm permits the assessment of individual differences for a novel ambiguity type (perceptual-lexical ambiguities) in a paradigm with demonstrated sensitivity to individual differences in lexical processing. An interference effect in the immediate condition would suggest that multiple activation occurs even given a constraining sentence context. These results would suggest that sentence context is not used directly to interpret the speech signal; rather, a perceptual-lexical ambiguity is resolved after both interpretations of the input are computed. A delay condition was employed in order to assess resolution of the ambiguity. A delay of $850 \mathrm{msec}$ was selected on the basis of previous work that demonstrated resolution of perceptual-lexical ambiguities by approximately $1 \mathrm{sec}$ (Connine, Blasko, \& Hall, 1991). Individual differences were assessed via a running memory span test. In the span test, subjects are required to remember the final word in a list of unrelated sentences, where the list length can vary from two to six sentences. An auditory version of the running memory span test was used. Individual differences in span size are assumed to reflect differences in working memory capacity for language (Just \& Carpenter, 1992; see also Daneman \& Carpenter, 1980). Individual differences in working memory capacity may be evident under circumstances that require the maintenance of more than one lexical hypothesis.

\section{EXPERIMENT 4}

\section{Method}

Subjects. A total of 56 subjects participated in the experiment for partial fulfillment of requirements in an introduction to psychology course The subjects were selected on the basis of performance on an auditory memory span task (28 were high-memoryspan subjects and 28 were low-memory-span subjects). No subjects reported any auditory deficits.

Memory span pretest. Prior to participation in the experiment, the subjects participated in an auditory memory span pretest. Auditory lists of unrelated sentences that were 13 to 16 words in length were created. Each sentence ended with a different word. The number of sentences in a given list varied from two to six sentences per set. Five sets at each list length were used. The sentences were recorded on audio tape, low-pass filtered $(4.8 \mathrm{kHz})$, digitized at $10 \mathrm{kHz}$ (12-bit resolution), and stored on disk for presentation to subjects. The subjects were instructed to listen to each of the sentences in a list over headphones and remember the last word of each sentence. After the final sentence in a list was presented, a recall cue was presented on the CRT screen (REPEAT FINAL WORDS). The subjects were instructed to write down the final word in each sentence in the just-heard list. On the basis of recall accuracy in a given list length, all subjects were classified into one of three groups: High span (4.0 or greater), medium span $(3.0,3.5)$, or low span $(2.5$ or lower). A total of 130 subjects participated in the auditory memory span pretest, and only those subjects who scored in the high-span or low-span regions participated in the main experiment.

Materials. The 20 perceptual-lexical ambiguities from the previous experiments were used. A sentence context was constructed for each stimulus that was more consistent with one interpretation of the ambiguity (see Appendix C). Sentences were constructed on the basis of the experimenters' intuitions, and the sentence bias was confirmed in a norming experiment. Half of the sentences were biased toward the voiced alternative, and half of the sentences were biased toward the voiceless alternative. The 20 sentences were recorded by the same talker used in the previous experiments, low-pass filtered $(4.8 \mathrm{kHz})$, digitized $(10 \mathrm{kHz}, 12-\mathrm{bit}$ resolution), and stored on disk. A waveform editor was used to digitally append an appropriate sentence context to a perceptuallexical ambiguity.

For control sentences, a synonym was selected for each perceptual-lexical ambiguity that matched the semantic bias of the appropriate sentence ${ }^{2}$ (see Appendix C). The synonyms were recorded with their appropriate sentence context, digitized, and stored on disk. A timing mark, inaudible to the subjects, was 
placed at the offset (immediate condition) or $850 \mathrm{msec}$ subsequent to the offset (delay condition) of the perceptual-lexical ambiguities and the control words. The timing mark was used to present the visual target and to time responses.

A set of 20 visual targets was selected from the set of related visual targets used in the previous experiments (see Appendix C). A visual target was selected for each sentence that was not related to the semantic bias of the sentence (e.g., a voiced-related target was selected if the sentence bias favored the voiceless interpretation of the perceptual-lexical ambiguity).

Procedure. Four experimental lists were created. In any list, a given sentence context was presented with either the perceptuallexical ambiguity or its control. Across the four lists, a visual target was presented with its appropriate perceptual-lexical ambiguity or its control at offset or at 850 -msec delay. The four lists were appropriately counterbalanced for stimulus type (perceptuallexical ambiguity or control) and visual target presentation (immediate or delay). The sentences were embedded in a list of other sentences with a variety of syntactic structures. Trials with related and unrelated targets were counterbalanced in the four lists.

The subjects were instructed to listen to the sentences and judge whether a visual target presented after each sentence was related or unrelated to the just-heard sentence. The subjects were told to respond quickly and accurately on an appropriately marked response box.

\section{Results and Discussion}

Table 4 shows relatedness judgment reaction time for correct responses as a function of auditory stimulus type (ambiguous and unambiguous) and delay (immediate and 850-msec delay) for high- and low-span subjects. Overall, high-span subjects showed a larger interference effect ( $139 \mathrm{msec})$ than did the low-span subjects $(76 \mathrm{msec})$ in the immediate condition. High-span subjects showed a dramatic decrease in the interference effect in the delay condition (19 $\mathrm{msec})$, and the low-span subjects showed a comparable degree of interference $(77 \mathrm{msec})$.

This pattern of data is seen very clearly in Figure 1, where difference scores (relatedness judgment reaction time for perceptual-lexical ambiguities-unambiguous control) are shown as a function of memory span and delay condition.

A three-way ANOVA was conducted on reaction times for the relatedness judgments with span (high vs. low) as a between-subject variable and delay (immediate and delay) and stimulus type (ambiguous and unam-

Table 4

Relatedness Judgment Reaction Times (in Milliseconds) and Percent Correct for High- and Low-Span Groups as a Function of Auditory Stimulus (Ambiguous vs. Unambiguous Control) and Delay in Experiment 4

\begin{tabular}{|c|c|c|c|c|}
\hline \multirow[b]{3}{*}{ Condition } & \multicolumn{4}{|c|}{ Auditory Stimulus } \\
\hline & \multicolumn{2}{|c|}{ Ambiguous } & \multicolumn{2}{|c|}{ Unambiguous Control } \\
\hline & RT & $\%$ Correct & RT & $\%$ Correct \\
\hline \multicolumn{5}{|c|}{ High-Span Group } \\
\hline Immediate & 982 & 91 & 843 & 93 \\
\hline Delay & 891 & 99 & 872 & 99 \\
\hline \multicolumn{5}{|c|}{ Low-Span Group } \\
\hline Immediate & 1,076 & 89 & 1,000 & 93 \\
\hline Delay & 1,055 & 98 & 978 & 98 \\
\hline
\end{tabular}

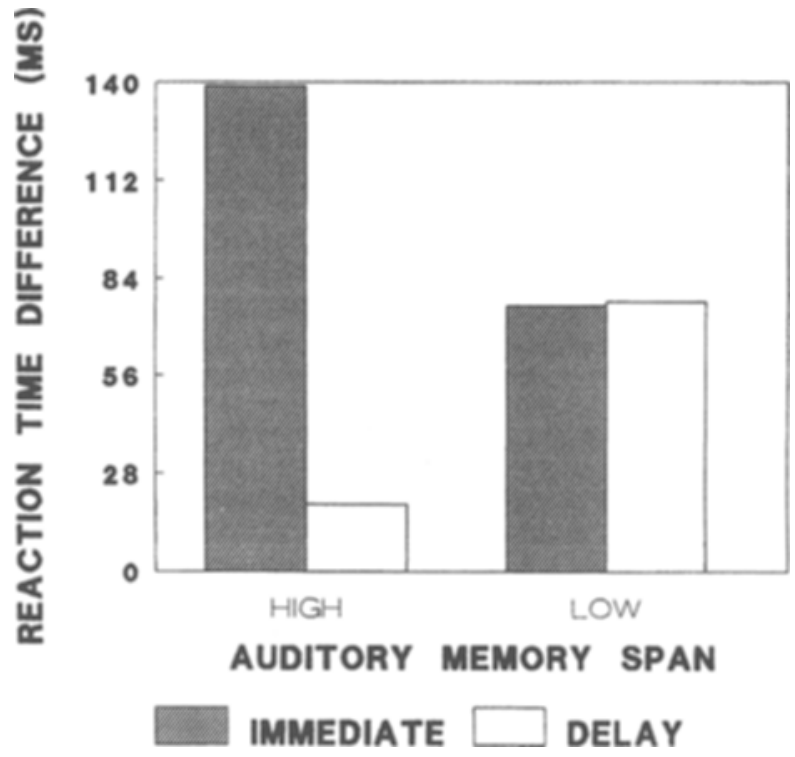

Figure 1. Reaction time difference (related vs. control) for highand low-memory-span subjects as a function of target delay (immediate and 850-msec delay) in Experiment 4.

biguous) as within-subject variables. The betweensubject main effect of span was significant $[F 1(1,54)=$ $7.87, M S_{\mathrm{e}}=119,995, p<.01 ; F 2(1,38)=23, M S_{\mathrm{e}}=$ $18,684, p<.01$ ], indicating faster reaction times for the high-span group of subjects. Overall, the main effect of stimulus type was significant, showing $78 \mathrm{msec}$ of interference for the ambiguous stimuli relative to the unambiguous stimuli $\left[F 1(1,54)=30, M S_{\mathrm{e}}=80, p<.01\right.$; $\left.F 2(1,38)=16, M S_{\mathrm{e}}=13,169, p<.01\right]$. The effect of stimulus type indicates that processing of the perceptuallexical ambiguities retained both potential interpretations (voiced and voiceless) of the perceptual-lexical ambiguities. The effect of stimulus type was modulated by both delay and span type. Overall, the degree of interference was greater in the immediate condition $(107 \mathrm{msec})$ than in the delay condition $(48 \mathrm{msec})$. The two-way interaction of stimulus type with delay was significant $\left[F 1(1,54)=4.5, M S_{\mathrm{e}}=11,430, p<.05\right.$; $\left.F 2(1,38)=4, M S_{\mathrm{e}}=9,228, p<.05\right]$. More importantly, the three-way interaction of span, delay, and stimulus type was significant $\left[F 1(1,54)=4.5, M S_{\mathrm{e}}=11,430\right.$, $\left.p<.05 ; F 2(1,38)=5, M S_{\mathrm{e}}=9,228, p<.05\right]$. The threeway interaction confirms the differential pattern of results for the high- and low-span groups depicted in Figure 1.3 To investigate the three-way interaction more fully, two additional $t$ tests were applied to the results of each memory span group. For the high-span subjects, there was a significant effect in the immediate condition $[t 1(27)=4.67, p<.01 ; t 2(19)=4.13, p<.01]$ but not in the delay condition $[t 1(27)=1.01, p=.32 ; t 2(19)=$ $0.43, p=.67]$. For the low-span subjects, there was a significant effect in the immediate condition $[t 1(27)=$ $4.67, p=.055 ; t 2(19)=4.13, p<.01]$. The delay con- 
dition showed a significant effect across subjects $[t 1(27)=$ $3.19, p<.01]$ that missed significance across items $[t 2(19)=1.81, p=.09]$.

Table 4 also shows percent correct as a function of auditory stimulus type and delay. A comparable ANOVA on percent correct data showed a main effect of delay $\left[F 1(1,54)=18.9, M S_{\mathrm{e}}=147, p<.01 ; F 2(1,38)=\right.$ $\left.12.8, M S_{\mathrm{e}}=375, p<.01\right]$, indicating that the subjects were more accurate in the delay condition ( $98 \%$ correct) than in the immediate condition ( $92 \%$ correct). No other effects were significant.

Two major patterns of data emerged when individual differences were considered. First, the immediate condition showed more effective multiple activation of the perceptual-lexical ambiguities for high-span subjects. Second, the delay condition showed more effective use of context to resolve the ambiguity for high-span subjects.

Consider, first, the findings in the immediate condition. If low-span subjects were unable to activate and maintain two lexical representations as effectively as the high-span subjects, then the low-span subjects would show a smaller interference effect that was due to a smaller portion of the subjects showing large interference effects. Closer inspection of the subject data indicate that the high-span subjects were considerably more consistent in showing interference effects. Overall, $82 \%$ of the high-span subjects and $57 \%$ of the low-span subjects showed interference effects. A $Z$ test for proportions showed that this difference was significant $(Z=$ $2.034, p<.05$ ). These data support the conclusion that the high-span subjects, as a group, were more consistent in activating and maintaining two lexical hypotheses than were the low-span subjects.

To further investigate the nature of the interference effects in the immediate condition, we examined separately the low- and high-span subjects that did show an interference effect. The interference effect found in the low-span subjects ( $220 \mathrm{msec}, S D=115$ ) was comparable to the interference effect found in the high-span subjects $(190 \mathrm{msec}, S D=126)$. This indicates that for those subjects showing evidence of multiple activation, the effectiveness of the activation was comparable across groups.

Consider, next, the pattern of results in the delay condition. The results for high-span subjects are quite straightforward in that effective multiple activation of lexical hypotheses was followed by effective use of contextual information to select the appropriate interpretation. The pattern of data for the high-span subjects is comparable to the findings of Gernsbacher and Faust (1991) for resolution of lexical ambiguity. Discussion of the results for low-span subjects in the delay condition is made somewhat less straightforward by the lack of consistent activation in the immediate condition. That is, it is nonsensical to discuss ambiguity resolution in the absence of effective multiple activation. Therefore, we conducted a subanalysis of the data that included only those low-span subjects who showed interference in the immediate condition.
Table 5

Relatedness Judgment Reaction Times (in Milliseconds) for the Low-Span Subjects Showing Interference in the Immediate Condition as a Function of Auditory Stimulus (Ambiguous vs. Unambiguous Control) and Delay in Experiment 4

\begin{tabular}{lcc}
\hline & \multicolumn{2}{c}{ Auditory Stimulus } \\
\cline { 2 - 3 } Condition & Ambiguous Control & Unambiguous Control \\
\hline Immediate & 1,144 & 924 \\
Delay & 1,056 & 971 \\
\hline
\end{tabular}

Table 5 shows the mean reaction time for the subgroup of low-span subjects as a function of stimulus type and delay. As previously described, the immediate condition showed an interference effect of $220 \mathrm{msec}(1,144$ vs. $924 \mathrm{msec})$. The delay condition also showed a sizeable interference effect of $85 \mathrm{msec}(1,056 \mathrm{vs} .971 \mathrm{msec})$. An ANOVA showed that the interference effect varied as a function of delay $[F(1,15)=6.25, p<.05]$. A $t$ test showed that the interference observed in the delay condition was significant $[t(15)=6.3, p<.05]$.

This analysis supports the claim that low-span subjects who could effectively activate and maintain multiple lexical hypotheses were less effective in using context to resolve the ambiguity than were high-span subjects.

The results of Experiment 4 suggest that activation of multiple lexical hypotheses operates without benefit from contextual information. In Fodor's (1983) terms, perceptual computations based on the speech signal are modular with respect to computation of sentence meaning. Elsewhere, we have suggested that modularity between early perceptual processes and sentence level representations is a functional architecture for language processing, since these representations are computed via different vocabularies (Connine, 1987). A second process involves commitment to a contextually appropriate interpretation in the face of ambiguity. Successful commitment to one lexical hypothesis by $850 \mathrm{msec}$ (the delay condition) was clearly evident for the high-span group. Low-span subjects showed evidence of inefficient suppression of the contextually inappropriate interpretation.

\section{GENERAL DISCUSSION}

Four experiments were reported that investigated processing of perceptual-lexical ambiguities. Experiments 1,2 , and 3 used methodological variants of the semantic cross-modal priming paradigm. These experiments demonstrated that listeners do activate multiple lexical hypotheses when processing a perceptual-lexical ambiguity. Experiment 4 extended these results to investigate the contribution of sentence context and individual differences to resolution of perceptual-lexical ambiguities. The results showed that individuals differ in the degree to which they are able to successfully activate and maintain multiple lexical hypotheses and in their ability to suppress the context-inappropriate lexical hypothesis. In 
addition, Experiment 4 showed that sentence context is used to select among equivalently available lexical hypotheses rather than as a direct source for lexical activation.

The results extend findings in speech perception concerning the recovery of phonetic features and spoken word processing. They demonstrate that the consequence of incomplete representations of speech categories for auditory word processing is not catastrophic - that is, ambiguity that results in competing specifications of a phonetic feature does not preclude the mapping of either feature to a compatible lexical representation. The experiments provide a means for linking the analysis of the speech signal to lexical processing, in particular, and to language processing, in general. An implicit assumption of much speech perception research is that unambiguous recovery of acoustic-phonetic information is necessary for successful speech perception. One possible basis for this implicit assumption is the additional (also implicit) assumption that an exhaustive recovery of information is necessary for other levels of spoken language processing. An immediate use for perceptual computations during spoken language comprehension is for the generation of lexical hypotheses. Our results show that lexical activation does not require computation of an exhaustive inventory of phonetic features that uniquely specify a particular speech segment. Given that the output of perceptual processes map onto lexical representations that do not require unambiguously specified information, it seems unnecessary to require speech segments to be unambiguously and completely represented.

This argument refocuses the emphasis in spoken word recognition on a view where ambiguity or incorrect information in the speech signal does not introduce a significant impediment to comprehension. An incomplete phonological representation can be tolerated at the level of speech sounds via a probabilistic representation at the level of phonetic features. A mapping of the output of perceptual processes to lexical representations based on goodness of fit permits a maintenance of ambiguous information at other processing levels. Notice that the relationship between perceptual processes and spoken word processing could have evolved so that a random commitment to a single lexical representation is made under conditions of ambiguity. Versions of the cohort model that rely on word-initial information to establish a set of lexical hypotheses (Marslen-Wilson \& Welsh, 1978; Marslen-Wilson \& Zwitserlood, 1989) could potentially accommodate processing of words with initial ambiguous phonemes by appealing to precisely this kind of mechanism. However, the resultant loss of information and potential garden path could have processing consequences for subsequent analyses. A commitment to a single lexical hypothesis (or its precursor phoneme hypothesis) given an ambiguous input would be incorrect some proportion of the time. In the case of a perceptual-lexical ambiguity, an incorrect commitment could occur as frequently as $50 \%$ of the time. This appears to be computationally expensive as well as incompatible with the present results. One positive conse- quence of late lexical commitment is that the language processing system is able to retain information that may be useful later. Late lexical commitment would be particularly useful when subsequent context provides clear semantic information concerning lexical identity (see Connine, Blasko, \& Hall, 1991). Connine, Blasko, and Hall demonstrated that commitment to one interpretation of a perceptual-lexical hypothesis was delayed by as much as about $1 \mathrm{sec}$ in the absence of prior contextual constraint. In general, lexical commitments in the absence of prior semantic context were made only when required by temporal pressures or syntactic considerations.

One purpose of Experiment 4 was to determine the nature of the relationship between spoken word recognition and the computations involved in higher levels of sentence comprehension. One possible outcome of Experiment 4 was that sentence context could activate only the semantically compatible alternative of the perceptuallexical ambiguities. This outcome would have been possible if sentence context influenced the weighting of perceptual information such that semantically compatible interpretations were given preference. This mechanism would constitute a direct influence of sentence context on auditory word recognition processes. In contrast, the results of Experiment 4 support a model where the mapping of acoustic-phonetic input to lexical representations are not directly modified by sentence level representations. As such, the findings are consistent with the conclusions using perceptual-lexical ambiguities in the phoneme identification task (Connine, 1987). These conclusions contrast with other work that has investigated the architectural relationship between lexical processing and sentence level representations. For example, Tabossi and colleagues (Tabossi, 1988; Tabossi, Colombo, \& Job, 1987) have shown that strongly biased sentence contexts were sufficient to result in activation of only one interpretation of a homonym. Tabossi argued that a sufficiently constraining sentence context can serve to selectively access the context appropriate interpretation. While the sentence contexts we used provided sufficient constraint for a clear preference between alternative interpretations of the perceptual-lexical ambiguity, the sentential constraint may not have been sufficient for providing activation to one word form. The sentence contexts used by Tabossi and colleagues focused on a salient feature of one interpretation of a homonym. Clearly, this was not the intent of the contexts we used, and alone may account for the different patterns of data. Alternatively, the contrasting conclusions for homonyms and perceptual-lexical ambiguities may lie in the nature of the ambiguous stimuli. Given that the likelihood of encountering lexical ambiguity is quite high in natural language, homonym representations may have evolved for efficient use of context via the delineation of maximally salient properties of alternative meanings stored with a single lexical form. Such a representation could provide an efficient link between information stored with a lexical entry and a developing semantic representation at the sentence level. Stated 
somewhat differently, processing of homonyms may be a special case of ambiguity where lexical representations are organized to capitalize on contextual constraints.

Lexical knowledge is another source of context information and effects of lexical status on phoneme perception are now well documented (Connine \& Clifton, 1987; Ganong, 1980; Pitt \& Samuel, 1993; Samuel, 1981). These results show that listeners identify a stimulus-initial ambiguous phoneme from a word-nonword speech continuum such that a word is formed. Although the mechanism underlying the influence of lexical status is controversial, these results minimally suggest that access to the lexicon is not denied by ambiguous information at word onset.

Considerable insight into processing of perceptuallexical ambiguities was derived from investigating individual differences in Experiment 4. The individual differences manipulation provided a window into processes involved in mapping the acoustic-phonetic input to a lexical representation and the use of sentence context. Experiment 4 demonstrated that activation of alternative lexical forms was more likely to remain for a longer period of time for low-span subjects than for high-span subjects. This finding mirrors the results of Gernsbacher and Faust (1991), who have shown that low comprehenders show similar perseveration of inappropriate homonym meanings. This aspect of the results indicates a parallel between processing homonyms and perceptuallexical ambiguities and implicates similar processes. The operative mechanism of suppression applies to alternative word meanings that map onto a single lexical form and extends to instances where two distinct word meanings map onto two different word forms. This parallelism provides a bridge between the two relatively separate literatures of spoken word recognition (computation of form) and lexical access (computation of meaning) and suggests a possible mechanism for resolving lexical form ambiguity. Recently, there has been an emphasis placed on the consequences of phonological similarity among words for recognition. A general finding that has emerged is that spoken words that sound similar to many words are less available than are spoken words that are relatively unique (Connine, 1994; Goldinger, Luce, \& Pisoni, 1989; Marslen-Wilson, 1990). Suppression may provide one means for accounting for the emergence of a single lexical candidate given multiple lexical hypotheses.

\section{REFERENCES}

ConNine, C. M. (1987). Constraints on interactive processes in auditory word recognition: The role of sentence context. Journal of Memory \& Language, 26, 527-538.

ConNine, C. M. (1994). Horizontal and vertical similarity in spoken word recognition. In C. Clifton, Jr., L. Frazier, \& K. Rayner (Eds.), Perspectives on sentence processing (pp. 107-120). Hillsdale, NJ: Erlbaum.

Connine, C. M., Blasko, D., \& Hall, M. (1991). Effects of subsequent context in auditory word recognition: Temporal and linguistic constraints. Journal of Memory \& Language, 30, 234-250.
Connine, C. M., Blasko, D., \& Titone, D. (1992, November). Acoustic-phonetic similarity in auditory word recognition: Vowels and consonants. Paper presented at the meeting of the Psychonomic Society, St. Louis.

Connine, C. M., Blasko, D., \& Titone, D. (1993). Do the beginnings of spoken words have a special status in auditory word recognition? Journal of Memory \& Language, 32, 193-210.

Connine, C. M., Blasko, D., Titone, D., \& Ferriby, J. (1993, April). Acoustic-phonetic similarity in auditory word recognition: Evidence from phoneme monitoring. Paper presented at the meeting of the Midwestern Psychological Association, Chicago.

ConNine, C. M., \& Clifton, C., JR. (1987). Interactive use of lexical information in speech perception. Journal of Experimental Psychology: Human Perception \& Performance, 13, 291-299.

Connine, C. M., Thtone, D., \& Blasko, D. (1991, November). Do the beginnings of auditory words have a special status? Paper presented at the meeting of the Psychonomic Society, San Francisco.

Connine, C. M., Titone, D., \& Ferriby, J. (1993, November). Vertical similarity in spoken word recognition. Paper presented at the meeting of the Psychonomic Society, Washington, DC.

DANEMAN, M., \& CARPENTER, P. (1980). Individual differences in working memory and reading. Journal of Verbal Learning \& Verbal Behavior, 19, 450-466.

EimAs, P. D., \& CoRBIT, J. D. (1973). Selective adaptation of linguistic feature detectors. Cognitive Psychology, 4, 99-109.

FODOR, J. (1983). The modularity of mind. Cambridge, MA: MIT Press.

FrancIs, W. N., \& KuČERA, H. (1982). Frequency analysis of English usage: Lexicon and grammar. Boston: Houghton Mifflin.

GANONG, W. F. (1980). Phonetic categorization in auditory word perception. Journal of Experimental Psychology: Human Perception \& Performance, 6, 110-125.

Garnes, S., \& Bond, Z. A. (1976). The relationship between semantic expectation and acoustic information. Phonologica, 3, 285-293.

GERNSBACHER, M. A. (1990). Language processing as structure building. Hillsdale, NJ: Erlbaum.

Gernsbacher, M. A., \& FAust, M. (1991). The mechanism of suppression: A component of general comprehension skill. Journal of Experimental Psychology: Learning, Memory, \& Cognition, 17, 245-262.

Goldinger, S., LuCE, P., \& Pisoni, D. B. (1989). Priming lexical neighbors of spoken words: Effects of competition and inhibition. Journal of Memory \& Language, 28, 501-518.

HAZAN, V., \& ROSEN, S. (1991). Individual variability in the perception of cues to place contrasts in initial stops. Perception \& Psychophysics, 49, 187-200.

JUST, M., \& CARPENTER, P. (1992). A capacity theory of comprehension: Individual differences in working memory. Psychological Review, 99, 122-149.

MarSLEN-WiLSON, W. (1987). Functional paralletism in spoken word recognition. Cognition, 25, 71-102.

MARSLEN-WiLSON, W. (1990). Activation, competition and frequency in lexical access. In G. T. M. Altmann (Ed.), Cognitive models of speech processing: Psycholinguistic and computational perspectives (pp. 148-172). Cambridge, MA: MIT Press.

MarSlen-Wilson, W., \& Welsh, A. (1978). Processing interactions and lexical access during word recognition in continuous speech. Cognitive Psychology, 10, 29-63.

MARSLEN-Wilson, W., \& ZWITSERLood, P. (1989). Accessing spoken words: On the importance of word onsets. Journal of Experimental Psychology: Human Perception \& Performance, 15, 576-585.

MASSARO, D. (1987). Speech perception by ear and eye: A paradigm for psychological inquiry. Hillsdale, $\mathrm{NJ}$ : Erlbaum.

MCClelland, J., \& Elman, J. (1986). The TRACE model of speech perception. Cognitive Psychology, 18, 1-86.

Milburg, W., Blumstein, S., \& Dworetzky, B. (1988). Phonological factors in lexical access: Evidence from an auditory lexical decision task. Bulletin of the Psychonomic Society, 26, 305-308.

Miller, J. L., Connine, C. M., Schermer, T., \& Kluender, K. (1983). A possible auditory basis for the internal structure of phonetic categories. Journal of the Acoustical Society of America, 73, 2124-2133.

Miller, J. L., \& Volaitis, L. (1989). Effect of speaking rate on the 
perceptual structure of a phonetic category. Perception \& Psychophysics, 46, 505-512.

NoRRIS, D. (1990). A dynamic-net model of human speech recognition. In G. T. M. Altmann (Ed.), Cognitive models of speech processing: Psycholinguistic and computational perspectives (pp. 87104). Cambridge, MA: MIT Press.

PitT, M., \& Samuel, A. (1993). An empirical and meta-analytic evaluation of the phoneme identification task. Journal of Experimental Psychology: Human Perception \& Performance, 19, 699725 .

Samuel, A. (1981). Phonemic restoration: Insights from a new methodology. Journal of Experimental Psychology: General, 110, 474-494.

SAmuel, A. (1982). Phonetic prototypes. Perception \& Psychophysics, 31, 307-314.

TABOSSI, P. (1988). Accessing lexical ambiguity in different types of sentential contexts. Journal of Memory \& Language, 27, 324340 .

Tabossi, P., Colombo, L., \& Job, R. (1987). Accessing lexical ambiguity: Effects of context and dominance. Psychological Research, 49, 161-167.

\section{NOTES}

1. An initial ANOVA with noise versus no noise added as a factor showed no difference among the two ambiguity conditions. Since this variable did not modulate the priming effect in any of the experiments, all reported analyses collapsed across the noise/no-noise conditions.

2. An alternative design would be to have control words that were perceptual-lexical ambiguities. Such a design would require that sentences designed to bias one interpretation of the experimental perceptual-lexical ambiguity were also consistent and sensible with both interpretations of an ambiguous control. Furthermore, the set of available ambiguous controls would have to simultaneously satisfy other lexical matching constraints, such as number of syllables, number of phonemes, and the relative frequency of the two possible interpretations. These constraints were impossible to satisfy simultaneously using the ambiguous auditory control design.

3. One possible alternative explanation is that the interference effects are simply due to the presence of the ambiguity per se rather than multiple lexical activation. A detailed examination of the pattern of interference effects renders such an explanation rather implausible. It seems highly unlikely that the mere presence of a perceptual-lexical ambiguity would produce interference effects of the magnitude found in the experiment (up to $139 \mathrm{msec}$ ). Furthermore, an ambiguity explanation would seem to predict that the interference effect would diminish with delay for all subjects. In contrast, low-span subjects showed comparable effects for both immediate and delay conditions. Finally, a simple interference effect explanation would be more consistent with data showing comparable interference effects in the immediate condition for all subjects. This contrasts with the asymmetry in interference effects for low- and high-span subjects.

APPENDIX A

VOT Values (in Milliseconds) for Each of the Speech Continua Used in the Experiments, and Percentage Voiced Response (\%VR) From the Identification Pretest Described in Experiment 1

\begin{tabular}{|c|c|c|c|c|c|c|c|c|c|c|c|c|c|c|}
\hline \multirow{3}{*}{$\begin{array}{l}\text { Perceptual-Lexical } \\
\text { Ambiguities } \\
\text { DIP-TIP }\end{array}$} & \multicolumn{12}{|c|}{ Identification Functions } & \multicolumn{2}{|c|}{ Related Targets } \\
\hline & \multicolumn{2}{|c|}{ VOT \%VR } & \multirow{2}{*}{$\frac{\text { VOT }}{\underline{40}}$} & \multirow{2}{*}{$\frac{\% \text { VR }}{40}$} & \multirow{2}{*}{$\frac{\text { VOT }}{43}$} & \multirow{2}{*}{$\frac{\% \text { VR }}{0}$} & \multicolumn{2}{|c|}{ VOT \%VR } & \multicolumn{2}{|c|}{ VOT \%VR } & \multirow{2}{*}{$\frac{\text { VOT }}{88}$} & $\%$ VR & \multirow{2}{*}{$\frac{\text { Voiced }}{\text { swIM }}$} & \multirow{2}{*}{$\begin{array}{l}\text { Voiceless } \\
\text { TOP }\end{array}$} \\
\hline & 13 & 80 & & & & & 45 & 0 & 48 & 0 & & 0 & & \\
\hline DIME-TIME & 0 & 100 & 30 & 80 & $\underline{35}$ & 40 & 40 & 0 & 45 & 0 & 90 & 0 & PENNY & CLOCK \\
\hline DART-TART & 0 & 100 & 40 & 40 & $\underline{43}$ & 40 & 45 & 40 & 48 & 0 & 75 & 0 & BOARD & SOUR \\
\hline $\mathrm{GAB}-\mathrm{CAB}$ & 2 & 100 & 45 & 60 & $\underline{48}$ & 60 & 52 & 60 & 56 & 20 & 70 & 0 & TALK & TAXI \\
\hline GLASS-CLASS & 0 & 100 & 59 & 100 & 62 & 80 & $\underline{68}$ & 60 & 71 & 60 & 90 & 20 & DRINK & ROOM \\
\hline GAIN-CANE & 7 & 100 & 45 & 60 & $\underline{53}$ & 50 & 57 & 20 & 61 & 20 & 85 & 20 & WEIGHT & LIMP \\
\hline GASH-CASH & 4 & 100 & 49 & 50 & $\underline{54}$ & 50 & 57 & 60 & 61 & 25 & 96 & 0 & CUT & MONEY \\
\hline GAUZE-CAUSE & 8 & 100 & 52 & 75 & 56 & 100 & $\underline{59}$ & 40 & 63 & 20 & 92 & 20 & BANDAGE & REASON \\
\hline BUSH-PUSH & 2 & 100 & 31 & 20 & 34 & 20 & $\underline{37}$ & 40 & 40 & 0 & 65 & 0 & PRESIDENT & PULL \\
\hline BEST-PEST & 0 & 100 & 25 & 60 & 28 & 40 & $\underline{31}$ & 50 & 34 & 40 & 61 & 0 & BETTER & BUG \\
\hline DUCK-TUCK & 0 & 80 & $\underline{40}$ & 60 & 42 & 20 & 45 & 20 & 47 & 0 & 70 & 0 & QUACK & FOLD \\
\hline DENSE-TENSE & 5 & 100 & 43 & 75 & 45 & 33 & $\underline{48}$ & 50 & 52 & 25 & 70 & 20 & THICK & NERVOUS \\
\hline GHOST-COAST & 0 & 100 & 45 & 60 & 49 & 80 & $\underline{52}$ & 40 & 55 & 20 & 75 & 0 & SPIRIT & BEACH \\
\hline GAUGE-CAGE & 11 & 100 & $\underline{48}$ & 40 & 51 & 0 & 55 & 0 & 59 & 20 & 88 & 20 & TIRE & BIRD \\
\hline GROUND-CROWNED & 15 & 100 & 73 & 100 & 77 & 75 & 79 & 80 & $\underline{81}$ & 60 & 98 & 20 & DIRT & QUEEN \\
\hline GREAT-CRATE & 7 & 100 & 78 & 60 & 81 & 60 & $\underline{83}$ & 40 & 85 & 60 & 100 & 20 & GRAND & воX \\
\hline GOLD-COLD & 0 & 100 & 45 & 100 & 49 & 80 & $\underline{55}$ & 50 & 59 & 40 & 95 & 0 & SILVER & нот \\
\hline GOOD-COULD & 5 & 100 & 49 & 60 & 53 & 60 & 57 & 20 & 61 & 20 & 85 & 20 & BAD & SHOULD \\
\hline BIG-PIG & 0 & 100 & 25 & 60 & $\underline{29}$ & 40 & 32 & 20 & 35 & 25 & 78 & 0 & LITTLE & HOG \\
\hline BACK-PACK & 5 & 100 & 28 & 80 & $\underline{31}$ & 40 & 34 & 60 & 37 & 40 & 70 & 0 & FRONT & TRAVEL \\
\hline
\end{tabular}

Note-Each underlined VOT value applies to the stimulus selected for use in the cross-modal priming experiments. 
APPENDIX B

The Unambiguous Auditory Controls (Experiment 2) and the Ambiguous Auditory Controls (Experiment 3)

\begin{tabular}{|c|c|c|c|c|}
\hline \multirow{3}{*}{$\begin{array}{l}\begin{array}{c}\text { Perceptual-Lexical } \\
\text { Ambiguity }\end{array} \\
\text { D/TIP } \\
\text { D/TIME }\end{array}$} & \multicolumn{2}{|c|}{$\begin{array}{l}\text { Unambiguous Auditory } \\
\text { Control } \\
\end{array}$} & \multicolumn{2}{|c|}{$\begin{array}{c}\text { Ambiguous Auditory } \\
\text { Control } \\
\end{array}$} \\
\hline & LAW & WALL & G/CLUE & (57) \\
\hline & CHORE & WAR & G/CLAD & $(72)$ \\
\hline D/TART & LINK & WINK & G/CROW & (68) \\
\hline $\mathrm{G} / \mathrm{CAB}$ & Loss & $\mathrm{RICH}$ & B/PUN & (42) \\
\hline G/CLASS & STEM & WHEN & B/PLANK & (44) \\
\hline G/CANE & RUSH & WASH & B/PAD & (28) \\
\hline G/CASH & MEN & ZONE & B/PARK & (30) \\
\hline G/CAUSE & SING & RING & B/POLE & (38) \\
\hline B/PUSH & SHAPE & RAG & $\mathrm{D} / \mathrm{TENT}$ & (48) \\
\hline B/PEST & SUNK & JUNK & D/TRIP & (84) \\
\hline $\mathrm{D} / \mathrm{TUCK}$ & RAW & MALL & G/CRIME & (84) \\
\hline D/TENSE & CHARM & WORM & G/CRASS & (78) \\
\hline G/COAST & RANK & ZINC & B/PATH & (48) \\
\hline G/CAGE & MOP & WHIP & B/PACK & (36) \\
\hline G/CROWNED & STORM & SPERM & B/PAIL & (28) \\
\hline G/CRATE & STALL & SCHOOL & $\mathrm{B} / \mathrm{PAY}$ & (36) \\
\hline $\mathrm{G} / \mathrm{COLD}$ & RAMP & RIDGE & B/PAT & (26) \\
\hline G/COULD & FALL & SANK & B/PAN & (22) \\
\hline $\mathrm{B} / \mathrm{PIG}$ & SHARE & RASH & D/TRAIN & (84) \\
\hline B/PACK & SHORE & LOVE & D/TORE & (31) \\
\hline
\end{tabular}

Note-The VOT values of the ambiguous auditory controls are shown in parentheses.

APPENDIX C

Sentence Contexts for Each Perceptual-Lexical Ambiguity, and the Auditory Control Words and Related Targets

Used in Experiment 4

\begin{tabular}{|c|c|c|c|}
\hline Ambiguity & Sentence Context & Auditory Control & Visual Target \\
\hline \multicolumn{4}{|c|}{ Voiceless Bias Contexts } \\
\hline DIP/TIP & LET'S CLIMB TO THE & ROOF & SWIM \\
\hline G/CANE & HE USED A & STICK & WEIGHT \\
\hline B/PUSH & HE TRIED TO & SHOVE & PRESIDENT \\
\hline D/TUCK & SHE MENDED THE & HEM & QUACK \\
\hline D/TENSE & THE ROPES WERE & TIGHT & THICK \\
\hline G/COAST & SHE DROVE TO THE & BEACH & SPIRIT \\
\hline G/CRATE & SHE OPENED THE & BOX & GOOD \\
\hline G/COULD & SHE KNEW SHE & WOULD & BAD \\
\hline B/PIG & HE BOUGHT THE & HOG & TALL \\
\hline B/PACK & HE LOADED THE & $\mathbf{B A G}$ & FRONT \\
\hline \multicolumn{4}{|c|}{ Voiced Bias Contexts } \\
\hline D/TIME & SHE FOUND A & PENNY & CLOCK \\
\hline D/TART & HE THREW THE & BALL & SOUR \\
\hline $\mathrm{G} / \mathrm{CAB}$ & SHE LIKED TO & TALK & TAXI \\
\hline G/CLASS & HE BROKE THE & CUP & TEACHER \\
\hline G/CASH & HE GOT A & CUT & MONEY \\
\hline G/CAUSE & HE WRAPPED THE & BANDAGE & REASON \\
\hline B/PEST & HE BOUGHT THE & FINEST & BUG \\
\hline G/CAGE & HE READ THE & METER & BIRD \\
\hline G/CROWNED & SHE DUG UP THE & YARD & QUEEN \\
\hline G/COLD & HE LOOKED FOR THE & SILVER & HOT \\
\hline
\end{tabular}

(Manuscript received August 13, 1993;

revision accepted for publication June 22, 1994.) 